\title{
Team Exercise: How Parenting Styles Affect The Next Generation
}

\author{
Kimberly Eddleston (Northeastern University) \\ Isabel C Botero (University of Louisville)
}

KEYWORDS: Leadership, Teaching Methods, Family

Business.

Because the family and business are inextricably intertwined in family businesses, parenting plays a critical role in shaping the next generation and their contribution to the family business. Parents influence whether their children are willing to (1) work in the family business (Jaskiewicz et al., 2015), (2) serve as successors (McMullen \& Warnick, 2015), and (3) whether a smooth succession occurs (Cater et al., 2016). Parents also affect the behaviors and career choices of their children (Young \& Friesen, 1992), particularly for those of business-owning families because the parent-child relationship transfers to the family business. As such, it is important to understand how different styles of parenting shape the next generation and ultimately influence the functioning and performance of family businesses.

Family psychologists identify four parenting styles based on the degree of support and control parents provide (i.e., authoritarian, authoritative, permissive, and neglectful) (Baumrind, 1967).

\section{low control high control}

$\begin{array}{lll}\text { high support } & \text { permissive } & \text { authoritative } \\ \text { low support } & \text { neglectful } & \text { authoritarian }\end{array}$

However, there is little understanding as to how these parenting styles influence the dynamics that develop within family businesses. In this exercise, teams of students are required to research a parenting style and provide examples of such parent-child interactions based on TV programs or movies. Each team is also required to explore how their assigned parenting style likely transfers to and manifests in a family business.

This exercise has been successfully used with undergraduate students studying family business as well as students enrolled in a course designed for next generation family business members.

\section{The Project}

This experiential exercise is designed to enhance student learning about the four parenting styles, how they influence the way individuals develop relationships with others, and, in turn, how these behaviors can affect the dynamics within a family business. The exercise is designed to be used in family business courses or workshops when discussing family factors and how they affect the behaviors of next generation members, and ultimately influence the family business. An example of a student presentation can be found below.

\section{Advance Preparation}

Students should be given at least two to three weeks to complete the team project. In classes of 25 students or less, students should be separated into four groups of 3 to 5 members each - one for each parenting style. With larger classes, the instructor can either have each parenting style presented by two or more groups or offer another project for the remaining student teams. With a total of four team presentations, usually 1 or 2 class periods are required depending on the length of the class. This gives all students the opportunity to deliver their presentation and attend all other presentations in the course. The directions for this project are below and
Copyright ( $) 2021$ The Authors. Entrepreneur \& Innovation Exchange is published at EIX.org. This is an open access article under the terms of the Creative Commons Attribution-NoDerivs License, which permits use and distribution in any medium, provided the original work is properly cited and no modifications or adaptations are made. View EIX.org Authorship Terms at https://eix.org/terms
FamilyBusiness 
can be downloaded as a PDF to share with students.

\section{Approximate Timing}

There are several components for the timing of the project:

- After teams are formed, the explanation of the project will take between 5 and 10 minutes.

- Presentations will take between 15 and 20 minutes per team.

- The discussion of the presentations can either be combined or after each presentation. If done after each presentation, the discussion usually lasts 5 to 10 minutes.

- The discussion will be led by the instructor and involve all class members

\section{Directions for Students Objective:}

The purpose of this assignment is for student teams to learn more about the different styles of parenting, what they mean for parent-child and sibling relationships, and how, in turn, these parenting styles are likely to extend to family relationships within a family business. Each team will research a different parenting style, find examples from film and/or TV series that reflect the parenting style, and then present their findings and recommendations to the class. Instructors will randomly assign a parenting style to each student team.

Each presentation should be 15 - 20 minutes, including the use of film and/or TV clips. Teams should provide an educational overview of their parenting style, explain how it manifests in families, and then extend their findings to the family business context. Film and/or TV clips should be used to demonstrate patterns of behavior and interaction that reflect the parenting style and possible outcomes that result. Presentations should end with advice for family business leaders; these can include advice for downplaying negative outcomes from a parenting style, realistic strategies to alter a damaging parenting style, how to lessen the transfer of negative family relationships to the family business, and/or how to capitalize on positive parenting components/ parenting styles in a family business.

\section{Components of the Presentation:}

Each presentation should be 15 to 20 minutes long.

Teams should:
1. Provide an educational overview of their assigned parenting style

2. Explain how it manifests in families

3. Extend their findings to the family business context

4. Provide advice to family business leaders about:

- How to downplay the negative outcomes from a parenting style.

- Realistic strategies to alter a damaging parenting style.

- How to lessen the transfer of negative family relationships to the family business.

- How to capitalize on positive parenting components/ parenting styles in a family business.

- Include Film and/or TV clips that demonstrate patterns of behavior and interaction that reflect the parenting style and possible outcomes that result from it. These clips should be between 30 seconds and 1 minute, and should show the parenting style within family interactions

\section{Deliverables}

- A Grading Rubric for the presentation can also be downloaded as a PDF. (Available above)

- Short paper that provides overview of the presentation and includes references embedded in the text, APA style. (3-5 pages)

- List of references (additional page)

\section{Discussion}

This project is a great way for students to explore the important role parents play in shaping the next generation. It pushes students to critically think about how relationship patterns are formed in families that then transfer to the business, for better or worse. It also encourages them to consider how different parenting styles contribute to the next generation's performance working in their family business. In turn, students are encouraged to reflect on how positive outcomes associated with a parenting style can be capitalized upon in the family business setting while negative outcomes are prevented or minimized.

\section{Variations for the Assignment}

- An alternative option for this project is to have role play scenarios of parent-child dyads instead 
of videos or have a combination of role play and videos.

- An interesting variation to add to this project is how parenting styles can manifest in different cultural backgrounds. For example, how do these parenting styles manifest in patriarchal cultures, or cultures with different deference to authority?

\section{Recommended reading available online to accompany project}

4 Types of Parenting Styles and Their Effects https://www.parentingforbrain.com/4-baumrind-

parenting-styles/

\section{Recommended academic readings:}

Chao, R. K. 2001. Extending research on the consequences of parenting style for Chinese Americans and European Americans. Child Development, 72(6): 1832-1843.

Combs, J. G., Shanine, K. K., Burrows, S., Allen, J. S., \& Pounds, T. W. 2020. What do we know about business families? Setting the stage for leveraging family science theories. Family Business Review, 33(1): 38-63.

Cramer, P. 2011. Young adult narcissism: A 20-year longitudinal study of the contribution of parenting styles, preschool precursors of narcissism, and denial. Journal of Research in Personality, 45(1), 19-28.

Givertz, M., \& Segrin, C. 2014. The association between overinvolved parenting and young adults' self-efficacy, psychological entitlement, and family communication. Communication Research, 41(8): 1111-1136.

McMullen, J. S., \& Warnick, B. J. 2015. To nurture or groom? The parent-founder succession dilemma. Entrepreneurship Theory and Practice, 39(6): 1379-1412.

Segrin, C., Woszidlo, A., Givertz, M., Bauer, A., \& Taylor Murphy, M. 2012. The association between overparenting, parent-child communication, and entitlement and adaptive traits in adult children. Family Relations, 61(2): 237-252.

Sorkhabi, N., \& Mandara, J. (2013). Are the effects of Baumrind's parenting styles culturally specific or culturally equivalent? In R. E. Larzelere, A. S. Morris, \& A. W. Harrist (Eds.), Authoritative parenting: Synthesizing nurturance and discipline for optimal child development (p. 113-135). American Psychological Association. $\quad$ https://doi.org/10.1037/13948-006 (https://psycnet.apa.org/doi/10.1037/13948-006) 


\section{Grading Rubric Presentation}

Group:

\section{Components of Project}

Content of the Presentation

Delivery of Presentation

Presentation Aids

Cohesiveness as Group during

Presentation

\section{Total}

Possible Points

\section{Points Obtained}

\section{Meets Expectations Exceeds Expectations}

\section{Content of Presentation}

Analysis of theThe presentation doesPresenters provide aPresenters provide in-Presenters provide an Learning Style not reflect any analysissuperficial analysis ofdepth analysis of somein-depth analysis of the of the parenting style. Itthe parenting style thataspects of theparenting style and reflects the interests ofis not clearly connectedparenting style.connect it well with the speakers. to the examples atHowever, this analysistheir examples.

hand. is not comprehensive and inclusive of all of the components of this parenting style.

Explanation of itsThere is no explicitPresenters identifyPresenters identify thePresenters provide a manifestation inexplanation of howsome of the waysways parenting stylesclear explanation of families parenting stylesparenting styles areare manifested inhow parenting styles manifest in the family. manifested in families,families, but this is notmanifest in the family, but these are not clear. done effectively. they identify clearly how each of the characteristics are present in a family and describe how it affects the dynamics of the family. 
Manifestation of The presenters do notPresenters identifyThe presenters makeThe presenters provide Learning Style in theexplain how parentingsome but not all thesuggestions about howa clear articulation of Family Businessstyles are visible indifferent ways thatparenting styleshow parenting styles Context family businesses. parenting styles aremanifest in the familymanifest in the family visible in familybusiness. However,business in the long businesses. they provide limitedand short-term run. articulation for howThey identify these styles are visiblechallenges and in a family firm. opportunities that are evident based on the style chosen.

Completeness andFails to address keyAddresses most of theAddresses each areaThoroughly addresses comprehensiveness components of theareas of the project. of the project to someeach area of the project.

extent. Providesproject. Identifies and Provides basicadequate coverage ofcomments on Provides limitedcoverage of relevantthe parenting styles. significant and relevant coverage of relevantissues related to the issues related to each issues related to theparenting styles. parenting style. part of the project.

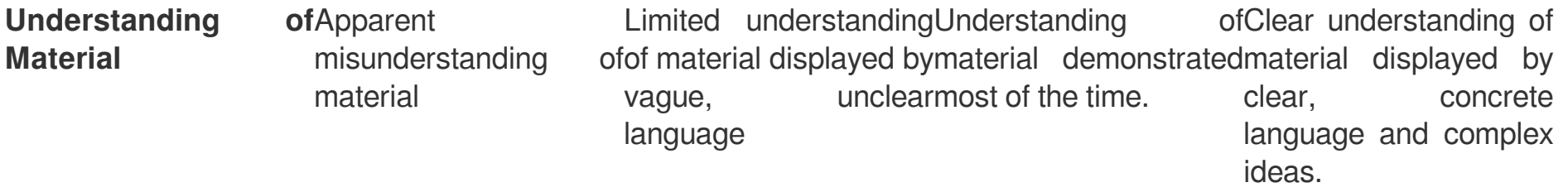

Meaningful Ideas are unclearUnelaborated ideasDepth of thoughtDepth and complexity application in theand/or not well-that are not fullysupported by selectionof thought supported by use of videos developed. Poor use ofexplained or supported, and contextualization ofrich, pertinent details; videos repetitive details.videos. supporting evidence Videos are not well (i.e., videos) leads to selected. high-level idea development

Support/Evidence forLittle or no data toSome evidence isMany details supportArgument is clearly Claims support the mainprovided, butthe argument but somesupported by accurate ideas/argument. information is not fullyare not fully explained.evidence that is Information used is notexplained, relevant toSome evidence is notcredible by the accurate. arguments or credible.relevant.

Missing important evidence.

audience. Sufficient details are provided to support the main points presented.

\section{Delivery}

Clarity

Explanation

ofNo apparent logicalContent is looselySequence ofDevelopment of ideas order of presentation,connected, transitionsinformation is well-in presentation are unclear focus. lack clarity organized for the mostclear through use of part; more clarity withspecific and 
transitions is needed appropriate examples, transitions are clear and create a succinct and even flow

Verbal componentsSpeakers are unable toSpeakers seemSome degree ofSpeakers are fluent of the presentation deliver the presentationuncomfortable. Severalnervousness coherently.

problems withapparent.
language $\quad$ usage.problems
Presenters speak toolanguage
slow or too fast, tooPresenters
loud or too soft, andproblems but
with many fillers. $\quad \begin{aligned} & \text { throughout } \\ & \text { presentation. }\end{aligned}$
isand poised. They use Minorlanguage comfortably withand appropriately for usage.the audience and havesituation. They speak adaptat an effective rate and thevolume, with very few fillers.

Non-Verba Non-verbal

Speakers are gesturingSpeaker gesturing tooSpeakers use gestures components of thecomponents of thetoo much or too little.much or too little,comfortably, and in line presentation presentation distractThey use distractinghowever they adaptwith their presentation from the ability of thegestures, do not havethrough thestyles. Eye contact is audience to receive theenough eye contactpresentation. Eyeappropriate for the message. and use spacecontact varies greatly.audience. Use of inappropriately. The speakers are ableSpace is appropriate to use space well forfor the situation. most part.

\section{Adherence presentation guidelines}

Q\&A

\section{Presentation Aids}

Design

$$
\begin{aligned}
& \text { toGoes beyond the time } \\
& \text { limit, forgets to follow } \\
& \text { presentation } \\
& \text { guidelines, and misses } \\
& \text { parts of the project. }
\end{aligned}
$$

Stays within time limitPresenters adhere to and follows thethe guidelines of the guidelines. provide extra information.

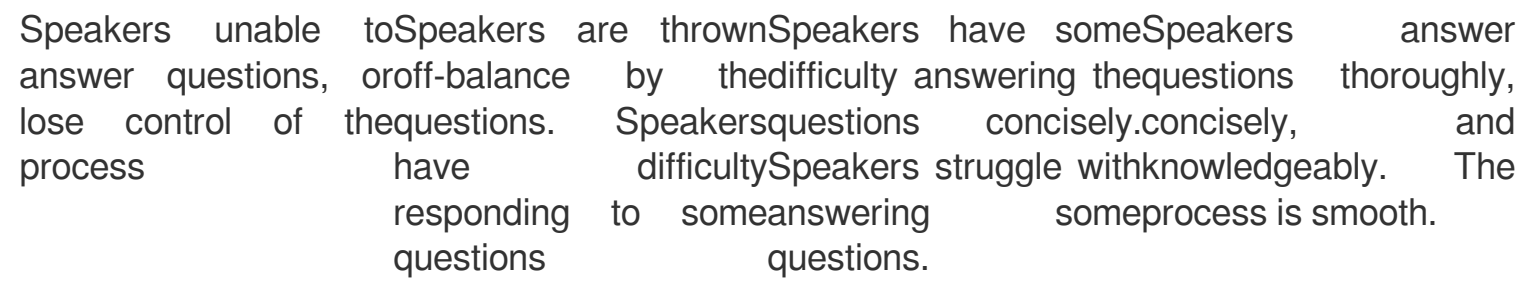

Speakers unable toSpeakers are thrownSpeakers have someSpeakers answer lose control of thequestions. Speakersquestions concisely.concisely, and process have difficultySpeakers struggle withknowledgeably. The questions questions. 
the audience cannot read. and follow.

Spelling \& Grammar Frequent errors inOccasional errors inMinor grammaticalNearly error-free which spelling, grammar, andgrammar punctuation punctuation, spelling anderrors and questionablereflects clear andword choices. Spellingunderstanding and has been proofed. thorough proofreading

Effective use ofPresents all of the

Presenters are able to information at the same time. Presenters read from slides. interact with the audience at the same time that they present information.

\section{Cohesiveness of Group}

Cohesiveness Group
ofPresenters areThere seems to bePresenters seem toPresenters work very disconnected fromsome connectionwork well together.well together. It seems each other, and do notbetween members. They understand eachlike they have practiced work as a group. However, there is veryother. However, theyand are able to work as little consideration forhave parts in whicha team. other group members. they show lack of practice as a team.

